\title{
Clinico- Radiologic and Genetic Study of Fraser Syndrome: A Case Report
}

\author{
Sujayendra Kulkarni ${ }^{1}$, Ashwini H. ${ }^{1}$, Ashalata Mallapur ${ }^{2}$, Yasmeen Maniyar ${ }^{3}$ \\ ${ }^{1}$ Department of Central Research Laboratory (CRL) (Division of Human Genetics), S. Nijalingappa Medical College and Hangal Shree \\ Kumareshwara Hospital \& Research Center, Navanagar, Bagalkot, India \\ ${ }^{2}$ Department of Obstetrics and Gynecology, S. Nijalingappa Medical College and S. Nijalingappa Medical College and Hangal Shree \\ Kumareshwara Hospital \& Research Center, Navanagar, Bagalkot, India \\ ${ }^{3}$ Department of Pharmacology, S. Nijalingappa Medical College and S. Nijalingappa Medical College and Hangal shree Kumareshwara \\ Hospital \& Research Center, Navanagar, Bagalkot, India
}

Email address:

sujayendra.kulkarmi@gmail.com (S. Kulkarni)

\section{To cite this article:}

Sujayendra Kulkarni, Ashwini H., Ashalata Mallapur, Yasmeen Maniyar. Clinico- Radiologic and Genetic Study of Fraser Syndrome: A Case Report. American Journal of Pediatrics. Vol. 7, No. 3, 2021, pp. 126-129. doi: 10.11648/j.ajp.20210703.17

Received: June 14, 2021; Accepted: July 12, 2021; Published: July 27, 2021

\begin{abstract}
Cryptophthalmos syndrome or Fraser syndrome is a rare autosomal recessive disorder, characterized by major features such as cryptophthalmos, cutaneous syndactyly, malformation of the larynx, abnormal genitalia, craniofacial dysmorphism, orofacial clefting, mental retardation and musculoskeletal anomalies. It is inherited in an autosomal recessive pattern where both the parents must be possessing one defective allele for Fraser syndrome. Since both the parents are carriers for the Fraser syndrome, there is a chance for $25 \%$ of their children being affected, $50 \%$ of their children to be carriers and $25 \%$ of their children to be normal. A 22 year old pregnant woman with a family history of third degree consanguineous marriage was diagnosed to have a fetus with many of the above findings in her anomaly scan which was done to manage child birth and as it is crucial to get prenatal diagnosis early in the pregnancy. Parents decided to terminate the pregnancy. Soon after the abortus was expelled, cord blood was collected and subjected to Karyotype test. Karyotype analysis revealed the absence of a short sequence on chromosome number 4 in q arm at $4 \mathrm{q} 21$ region, which may be responsible for the above mentioned deformities. Due to the high morbidity and mortality associated with Fraser syndrome, early detection by amniotic fluid karyotyping may be helpful in early intervention.
\end{abstract}

Keywords: Cryptophthalmos Syndrome, Fraser Syndrome, Syndactyly

\section{Introduction}

Fraser syndrome is an autosomal recessive congenital disorder [1]. The initial tale of Fraser Syndrome is attributed to Zehender and coworkers in 1872, when it was known as 'cryptophthalmos syndrome', as reported by Khoury et al [2]. Fraser syndrome is named after the geneticist, George R. Fraser, who first described the syndrome in 1962. Considering this syndrome as a complex disorder with multiple anomalies, with or without cryptophthalmos, Fraser syndrome is the preferred terminology [3]. As per the diagnostic criteria in the opinion of Thomas et al. [6], Fraser syndrome is characterized by developmental defects, including cryptophthalmos (where the eyelids fail to separate in each eye) and malformations in the genitalia (suchas micropenis, cryptorchidism or clitoromegaly) [4]. Congenital malformations of the nose, ears, larynx and renal system, as well as mental retardation manifest occasionally. Syndactyly (fused fingers or toes) has also noted $[5,6]$. The reported incidence of Fraser Syndrome is 0.043 per 10,000 neonates and 1.1 in 10,000 stillbirths, worldwide [7]. The reported incidence of Fraser Syndrome is 0.043 per 10,000 live born infants and 1.1 in 10,000 stillbirths, worldwide [7].

Cryptophthalmos (hidden eye) refers to a group of rare congenital eyelid deformity, in which the eyelids fail to separate. Patients with a syndromic combination of acrofacial and urogenital malformations with or without cryptophthalmos should be taken into consideration for Fraser syndrome [8]. 
Subjecting amniotic fluid sample for karyotyping test between 16-20 weeks of gestation can help diagnosis and early intervention in cases of congenital malformations.

The genetic background of this disease has been linked to FRAS1, a gene involved in skin epithelial morphogenesis during early growth [9]. The FRAS1 gene is pin pointed on the long arm of chromosome 4 (at $4 \mathrm{q} 21$ region). This disorder has also been coupled among FREM2 gene that originate in chromosome 13 [10] and with mutations in GRIP1 gene of chromosome 12 [11]. FRAS1 gene is responsible for proper development of skin, internal organs and tissues. Protein from the gene GRIP1 is responsible for the proteins from the genes FRAS1 and FREM2 to get to the actual location in the cell to work together [12]. The proper identification of Fraser syndrome can be accurately made after birth based on its distinctive association of malformations. Occasionally, it is possible to establish this disorder due to abnormal prenatal ultrasonographic features such as, polyhydramnios or oligohydramnios, echogenic lungs and renal abnormalities/agenesis [1].

\section{Case Report}

A 22 year old pregnant woman came with a history of previous spontaneous abortion at 12 weeks of gestation and wanted to undergo anomaly scan for the present pregnancy. She had obstetric history of gravida 2, para 0 , abortion 1 , living 0 . Family history revealed second degree consanguineous marriage. Her first trimester was uneventfulshe had regular antenatal checkups and was supplemented with folic acid, iron and other general nutritional supplementations. Nuchal translucency scan was normal.

Second trimester was uneventful, further she underwent anomaly scan at 24 weeks of gestation. Anomaly scan report at 24 weeks of gestation revealed the following findings suggestive of abnormal thorax, abnormal abdomen, abnormal kidney and urinary bladder, and abnormal extremities in the fetus:

1) The lungs were massively enlarged and hyperechogenic, resulting in compression of the heart, and development of ascites.

2) The bronchial tree and trachea were dilated and the diaphragm inverted.

3) Abdomen was distended with massive ascites.

4) Bilateral club foot.

5) Chronic high airway obstruction syndrome.

6) The bladder was small and cystic dysplasia of the kidneys was noted (Figure 1).

7) Cystic hygroma was noted in the fetus.

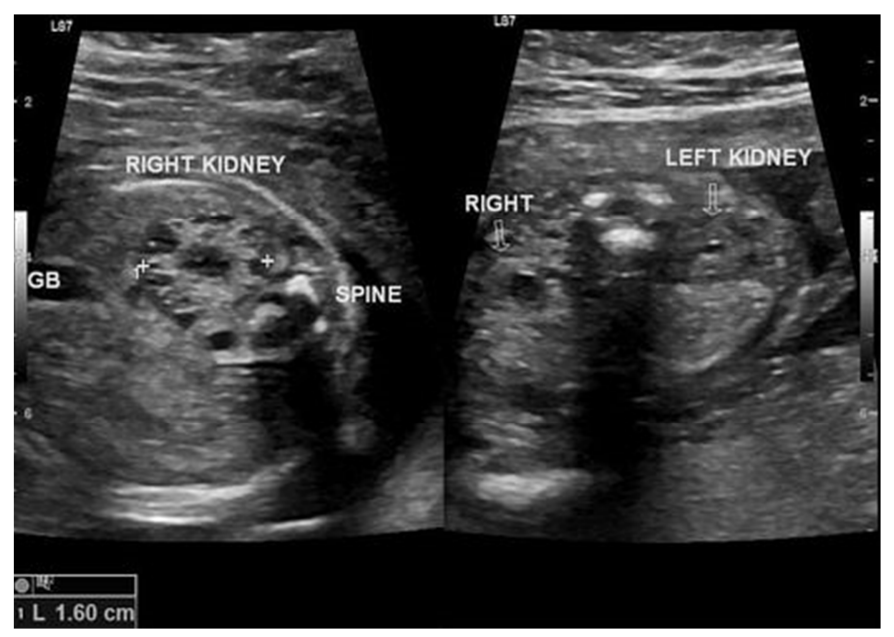

Figure 1. Ultrasonographic image of fetus, showing cystic dysplasia of kidneys.

Ultrsonography also revealed oligohydramnios. Due to the above mentioned anomalies, medical termination of pregnancy was done. On gross examination, the fetus showed grade 3 microtia of ears, saddle depression of dorsum of nose, syndactyly in fingers, cryptorchidism and micropenis and bilateral clubfoot (Figure 2).

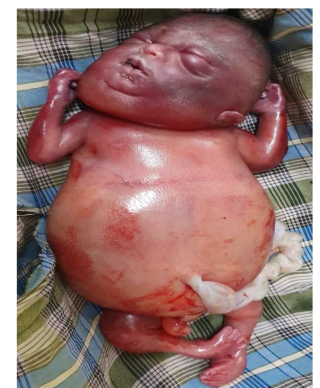

Figure 2. Fetus showing club foot, syndactyly, malformed ears and cryptopthalmous. 
Soon after termination of pregnancy, cord blood was collected and subjected to Karyotype analysis, using standard Karyotyping protocol. Karyotype of the sample revealed the absence of a short sequence on chromosme number 4 in q arm, at $4 \mathrm{q} 21$ region (Figure 3).
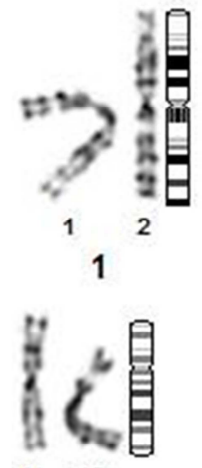

$11 \quad 12$ 6

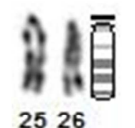

13
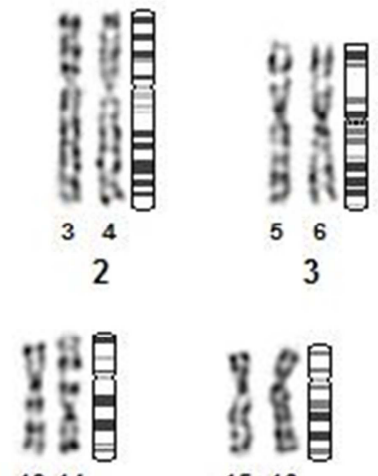

1314

7

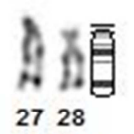

14

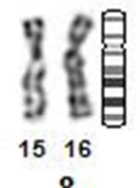

8

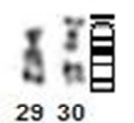

15

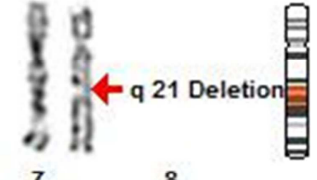

4

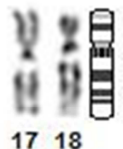

9

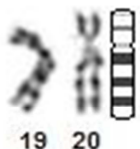

10

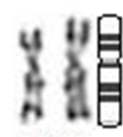

2122

11

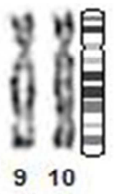

5

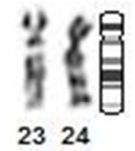

12

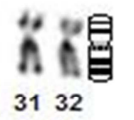

16

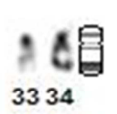

17

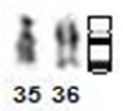

18

\begin{tabular}{|c|c|c|c|c|c|}
\hline 889 & × 89 & $A \& \bar{B}$ & : $6 \overline{\bar{B}}$ & $\pi$ & 48 \\
\hline 3738 & 3940 & 4142 & 4344 & 45 & 46 \\
\hline 19 & 20 & 21 & 22 & $x$ & $Y$ \\
\hline
\end{tabular}

Figure 3. Karyotype analysis of cord blood of abortus with Fraser syndrome showing deletion on one of the chromosome number 4 at $4 q 21$ region.

\section{Exchange of Views}

Fraser Syndrome is a rare genetic disorder with just more than 200 cases reported till date in the indexed literature [7]. It is a combination of acrofacial and urogenital malformation with cryptophthalmos. In 1986, Thomas et al laid down the diagnostic criteria for Fraser syndrome. Two major and one minor criterion or one major and at least four minor criteria are needed for the diagnosis [7].

Table 1. Diagnostic criteria.

\begin{tabular}{ll}
\hline Diagnostic criteria by Thomas et $\mathbf{a l} \cdot \mathbf{~ [ 7 ]}$ & Minor \\
\hline Major & 1. Congenital malformation of the nose \\
\hline 1. Cryptophthalmos & 2. Congenital malformation of the ears \\
2. Syndactyly & 3. Congenital malformation of the larynx \\
3. Abnormal genitalia & 4. Skeletal defects \\
4. Sibling with cryptophthalmos syndrome & 5. Umbilical hernia \\
& 6. Renal agenesis \\
& 7. Mental retardation \\
\hline
\end{tabular}

The findings in this case are compatible with the diagnosis of Fraser syndrome according to the major and minor criteria proposed by Thomas et al. [7]. In the present case, the major criteria were syndactyly and abnormal genitalia and the minor criteria were malformations of the nose, ears, skeletal defects and cystic dysplasia of the kidneys.

Syndactyly has been considered as a major feature of Fraser syndrome that occurs in $77 \%$ of the patients. Syndactyly is always cutaneous and in most cases involves fingers and toes [15]. In the present case, there was syndactyly in the fingers.

In males, according to AM Slavotinek, CJ Tifft et al [1], abnormal genitalia such as cryptorchidism - (31.5\%), micropenis, phimosis, chordee, hypospadias, and scrotal hypoplasia were noted [14]. In the present case, cryptorchidism and micropenis were noted.

Congenital malformation of the nose and ears has been considered as minor criteria. According to AM Slavotinek, CJ 
Tifft et al [1], nasal anomalies were common with having a broad nose or nasal bridge $(20.5 \%)$, depressed or flat nasal bridge $(11.1 \%)$, and bifid nose or a midline nasal groove $(15.4 \%)$. Coloboma of the nares or notched nares were existing $(11.1 \%)$. In the present case, there was saddle depression of dorsum of nose.

Malformed and/or low set ears (53.8\%), microtia (16.2\%), and atresia or stenosis of the external auditory meatus $(17.9 \%)$ were noted [14]. In the present case, there was grade 3 microtia of ears.

According to Peer et al [13] laryngeal stenosis or atresia was reported in $30.8 \%$. Choanal stenosis or atresia (76\%) and subglottic stenosis $(8.5 \%)$ were also described. In the present case lungs were massively enlarged and hyperechogenic resulting in compression of heart. The bronchial tree and trachea were dilated.

Skeletal defects has been considered as a minor feature of FS. According to AM Slavotinek, CJ Tifft et al [1] absence or hypoplasia of the orbital or skull bones $(10.2 \%)$ and defects in skull ossification $(6.8 \%)$ were among the most frequent musculoskeletal anomalies. Talipes $(8.5 \%)$ and abnormalities involving the pubic symphysis $(7.7 \%)$ were relatively common. In the present case, bilateral clubfoot was noted.

According to AM Slavotinek, CJ Tifft et al [1] out of 117 patients bilateral renal agenesis with or without agenesis of the ureters was present in $23.1 \%$ and unilateral renal agenesis with or without ureteral agenesis in $22.2 \%$. The bladder was small or absent in $17.1 \%$ and cystic dysplasia of the kidneys was reported in $12 \%$. In the present case, cystic dysplasia of the kidneys and small bladder was observed.

Fraser syndrome is an autosomal recessive inherited disorder. The parents of the affected children are sometimes but not always consanguineous. Consanguinity of marriage is reported in $15-24.8 \%$ of the cases $[14,15]$. Parental consanguinity was also noted in this case.

The clinically variable anomalies corresponding with Fraser syndrome support the genetic heterogeneity of the syndrome. The gene of the Fraser syndrome was localized to 4q21 FRAS1 gene [1]. In the present study also, deletion in $4 \mathrm{q} 21$ region was noted.

Fraser syndrome is otherwise known as cryptophthalmos syndrome. However, cryptophthalmos is not an essential component of this syndrome. A review of 87 cases by Thomas et al, showed the absence of cryptophthalmos in 14 cases [7]. According to R. Koenig and J. Sprancer et al [14] Fraser syndrome was noted without cryptophthalmos. In the present case also, there was absence of cryptophthalmos.

\section{Conclusion}

Fraser syndrome is an autosomal recessive disorder, and is associated with high mortality and morbidity. Hence, subjecting amniotic fluid for karyotyping between 16-20 weeks of gestation might confirm the presence or absence of the Fraser syndrome which may be helpful in early intervention.

\section{References}

[1] Slavotinek AM, Tifft CJ. Fraser syndrome and cryptophthalmos: A review of the diagnostic criteria and evidence of phenotypic modules. Journal of Medical Genetics; 2002; 39: 623-33.

[2] Khoury E, Golalipour MJ, Haidary K, Adidi B. Fraser or cryptophthalmos syndrome: A case report. Archives of Iranian Medicine 2004; 7: 307-9.

[3] Francannet C, Lefrançois P, Dechelotte P, Robert E, Malpuech G, Robert JM. Fraser syndrome with renal agenesis in two consanguineous Turkish families. American Journal of Medical Genetics 1990; 36 (4): 477-479.

[4] Van Haelst MM, Scambler PJ, Hennekam RC. Fraser syndrome: A clinical study of 59 cases and evaluation of diagnostic criteria. American Journal of Medical Genetics 2007; 143A (24): 3194-203. doi: 10.1002/ajmg.a.31951. PMID 18000968.

[5] Kalpana Kumari MK, Kamath S, Mysorekar VV, Nandini G. Fraser syndrome. Indian Journal of Pathology and Microbiology 2008; 51 (2): 228-9. doi: 10.4103/03774929.41664.

[6] Thomas IT, Frias JL, Felix V, Sanchez de Leon L, Hernandez RA, Jonen MC. Isolated and syndromic cryptophthalmos. Am J Med Genet 1986; 25: 85-90.

[7] Narang M, Kumar M, Shah D. Fraser-cryptophthalmos syndrome with colonic atresia. Indian Journal of Pediatrics. 2008; 75: 189-91.

[8] McGregor L, Makela V, Darling SM. Fraser syndrome and mouse blebbed phenotype caused by mutations in FRAS1/Fras1 encoding a putative extracellular matrix protein. Nature Genetics 2003; 34: 203-8.

[9] Jadeja S, Smyth I, Pitera JE. Identification of a new gene mutated in Fraser syndrome and mouse myelencephalic blebs. Nature Genetics 2015; 37: 520-525.

[10] Chanze D. Fraser syndrome due to mutations in GRIP1. A clinical phenotype in two families and expansion of the mutation spectrum. American Journal of Medical Genetics 2014; 164: 837-840.

[11] Petrou P, Makrygiannis AK, Chalepakis G. The Fras1/Frem family of extracellular matrix proteins: structure, function, and association with Fraser syndrome and the mouse bleb phenotype. Connect Tissue Res. 2008; 49 (3): 277-82.

[12] Berg C, Geipel A, Germer U. Prenatal detection of Fraser syndrome without cryptophthalmos: case report and review of the literature. Ultrasound Obstet Gynecol. 2001; 18: 76-80.

[13] Pe'er J, BenEzra D, Sela M, Hemo I. Cryptophthalmos syndrome. Clinical and histopathological findings. Ophthalmic Paediatric Genetics; 1987; 8: 177-82.

[14] Koenig R, Sprancer J. Cryptophthalmos - syndactyly syndrome without cryptophthalmos. Clinical Genetics 1986; 29: 413-416.

[15] Ramsing M, Rehder H, Holgreve W, Meinecke P, Lenz W. Fraser syndrome with Cryptophthalmos and syndactyly in the fetus and newborn. Clinical Genetics 1990; 37: 84-96. 